Dokuz Eylül Üniversitesi-Mühendislik Fakültesi

Fen ve Mühendislik Dergisi

Cilt 19 Sayı 55 Ocak 2017
Dokuz Eylul University-Faculty of Engineering Journal of Science and Engineering Volume 19 Issue 55 January 2017

DOI: $10.21205 /$ deufmd. 2017195521

\title{
The Effect of Bichromatic Potential on Thirring Instantons
}

\author{
Fatma AYDOGMUS*1
}

1İstanbul Üniversitesi, Fen Fakültesi, Fizik Bölümü, İstanbul

(Alınış / Received: 14.06.2016, Kabul / Accepted: 14.11.2016, Online Yayınlanma / Published Online: 09.01.2017)

\begin{abstract}
Anahtar Kelimeler Özet: Thirring model is a 2D conformally invariant pure fermionic Soliton, Instanton, Bichromatic, Nonlinear dynamic. model with no mass term. The model admits particle-like solutions and these solutions are instantonic in character. In this study, the effect of bichromatic potential on Thirring instantons is investigated to get a better idea of their nonlinear dynamical nature. For this purpose, the phase portraits are constructed. Under some parameter conditions, chaos is observed.
\end{abstract}

\section{Bikromatik Potansiyelin Thirring İnstantonları Üzerine Etkisi}

\section{Keywords}

Soliton,

Instanton,

Bikromatik,

Lineer olmayan

dinamik.

\begin{abstract}
Thirring model iki boyutlu konformal invaryant kütlesiz saf bir fermiyonik modeldir. Model parçacık benzeri çözümler sergiler ve bu çözümler instanton karakterindedir. Bu çalışmada Thirring instantonlarının lineer olmayan dinamiğini daha iyi kavrayabilmek için bikromatik potansiyelin model üzerindeki etkisi incelenmiştir. $\mathrm{Bu}$ amaçla faz portreleri inşa edilmiş ve bazı sistem parametre değerleri için kaos gözlemlenmiştir.
\end{abstract}

* Sorumlu Yazar: fatma.aydogmus@gmail.com, fatmaa@istanbul.edu.tr

\section{Introduction}

It is known that solitons play an important role in many areas, ranging from theoretical physics to applied mathematics. They were discovered as undissipated surface waves on water and realized to obey nonlinear wave equations [1-2]. Instanton, one of the leading soliton types, has a finite action with zero energy. Instantons are classical topological solutions of the field equations of any given model in quantum field theories which lie behind the standard model of particles. [2]. Such solutions were obtained in exact analytical form for a variety of models including Quantum Chromo Dynamics (QCD). They have been considered as configurations of quantum fields that provide a tunnelling effect between the vacuums that have different topologies in space-time [3-5]. The name indicates that the fluctuations are confined to an instant in space-time [6]. They originate from topological structure of vacuum in non-abelian gauge field theories [6-7]. Despite their undoubted importance for the theory of strong and electroweak interactions, direct experimental evidence for instanton-induced processes have being lacking until now. It is hoped that an attentive analysis of Large Hadron Collider (LHC) data in CERN might bring experimental confirmation of such processes [8-11]. 
Thirring model is a well-known system of single self-interacting massless fermions in $(1+1)$ space-time dimensions with the nonlinear self-interaction term [12]. The model is pure fermionic with a nonlinear coupling and it has conformal symmetry. It contains many of the typical features of the quantization of relativistic quantum field theories and plays a very fruitful role in the progress of field theory [12]. A class of spinor-type instanton solutions of conformally invariant pure spinor Thirring field equation was found by the spontaneous symmetry breaking of the conformal invariance $[12,13]$. We call these solutions as Thirring instantons. Recently, the role of the coupling constant in the evolution of the two dimensional spinor-type Thirring instantons has been investigated in phase space $[14,15]$. The vector analysis and orientations of Thirring instantons have been studied [16]. Also there have been a number of studies on instantons (See some of these in Refs. [17-21]).

In this paper, the regular and chaotic behaviours of the spinor-type Thirring instantons are studied under the bichromatic potential to get more information about the quantum dynamics of spinor type instantons in vacuum. We investigate the effects of the external potential in the long time behaviours of the spinor-type Thirring instantons in phase space. To this end, we consider the general dynamics of Thirring nonlinear differential equations system formed by the use of Heisenberg ansatz with the addition of a bichromatical potential.

\section{Model}

The Thirring wave equation is

$$
i \sigma_{\mu} \partial_{\mu} \psi+g(\bar{\psi} \psi) \psi=0
$$

Where $g$ is the positive coupling constant, $\sigma_{\mu}$ are Pauli matrices $(\mu=1,2,3)$ and the fermion field $\psi(x)$ has scale dimension $\frac{1}{2}$ [22].

The complex form of the Euclidian configuration of Heisenberg ansatz [23] is

$$
\psi=\left[i x_{\mu} \sigma_{\mu} \chi(s)+\varphi(s)\right] C
$$

with an arbitrary spinor constant $C$. $\chi(s)$ and $\varphi(s)$ are real functions of $s=x^{2}+t^{2}\left(x_{1} \equiv x, x_{2} \equiv t\right)$. Inserting Eq. (2) into Eq. (1), with

$$
i \sigma_{\mu} \partial_{\mu} \psi=\left[\begin{array}{l}
-2 \chi(s)-2 s \frac{\mathrm{d} \chi(s)}{\mathrm{d} s} \\
+2 i x_{\mu} \sigma_{\mu} \frac{\mathrm{d} \varphi(s)}{\mathrm{d} s}
\end{array}\right] \bar{C} C
$$

and

$$
g(\bar{\psi} \psi) \psi=\left[\begin{array}{l}
i g x_{\mu} \sigma_{\mu} \chi(s) \\
\left(s \chi^{2}(s)+\varphi^{2}(s)\right) \\
+g \varphi(s) \\
\left(s \chi^{2}(s)+\varphi^{2}(s)\right)^{\frac{1}{3}}
\end{array}\right]
$$

$(\bar{C} C) C$,

where

$$
\begin{aligned}
& (\bar{\psi} \psi)=\left(s \chi^{2}(s)+\varphi^{2}(s)\right) \\
& (\bar{C} C),
\end{aligned}
$$

one obtains the following nonlinear differential equations system

$$
\begin{aligned}
& 2 \chi(s)+2 s \frac{\mathrm{d} \chi(s)}{\mathrm{d} s}- \\
& g \varphi(s)\left[s \chi(s)^{2}+\varphi(s)^{2}\right] \\
& \bar{C} C=0
\end{aligned}
$$




$$
\begin{aligned}
& 2 \frac{\mathrm{d} \varphi(s)}{\mathrm{d} s}+ \\
& g \chi(s)\left[s \chi(s)^{2}+\varphi(s)^{2}\right]^{1 / 3} \\
& \bar{C} C=0 .
\end{aligned}
$$

$$
\begin{aligned}
& \begin{array}{l}
\text { Substituting } \quad \chi=A s^{-\sigma} F(u) \text { and } \\
\varphi=B s^{-\tau} G(u), \quad \text { with } u=\ln (s) \text { and }
\end{array} \\
& \sigma=\tau+\frac{1}{2}, \tau=\frac{1}{4} \text { and } A^{2}=B^{2}[12] . \\
& 2 A|s|^{-\sigma} F(u)-2 s \sigma A|s|^{-\sigma-1} F(u)+ \\
& 2 s A|s|^{-\sigma} F(u) \frac{1}{s}- \\
& g B|s|^{-\tau} G(u)\left(\begin{array}{l}
s A^{2}|s|^{-2 \sigma} F(u)^{2} \\
+B^{2}|s|^{-2 \tau} G(u)^{2}
\end{array}\right) \\
& (\bar{C} C)=0 \\
& -2 \tau B|s|^{-\tau-1} G(u)+2 B|s|^{-\tau} G(u) \frac{1}{s}+ \\
& g A|s|^{-\sigma} F(u)\left(\begin{array}{l}
s A^{2}|s|^{-2 \sigma} F(u)^{2} \\
+B^{2}|s|^{-2 \tau} G(u)^{2}
\end{array}\right) \\
& (\bar{C} C)=0,
\end{aligned}
$$

we achieve the dimensionless form of the non-linear ordinary coupled differential equations system (7) as

$$
\begin{aligned}
& 2 \frac{d F(u)}{d u}+\frac{1}{2} F(u) \\
& -\alpha A B\left(F(u)^{2}+G(u)^{2}\right) G(u)=0 \\
& 2 \frac{d G(u)}{d u}-\frac{1}{2} G(u) \\
& -\alpha A B\left(F(u)^{2}+G(u)^{2}\right) F(u)=0
\end{aligned}
$$

Here $\mathrm{F}$ and $\mathrm{G}$ are dimensionless functions of $\mathrm{u}, \alpha, \mathrm{A}$ and $\mathrm{B}$ are constant. The bichromatic potential is

$$
\begin{aligned}
& V=C_{1} \operatorname{Cos}^{2}\left(\omega_{1} u\right) \\
& +C_{2} \operatorname{Cos}^{2}\left(\omega_{2} u\right)
\end{aligned}
$$

Especially, this potential is one of the potentials used to trap bosons in BoseEinstein condensate. The bichromatic potential has two different frequencies.
This property is suitable for double-well conditions of instantons. Also more parameters are used in numerical process. If we redefine Thirring Nonlinear Differential Equations System with the bichromatic potential to get more information about the quantum dynamics of spinor type instantons as

$$
\begin{aligned}
& 2 \frac{d F(u)}{d u}+\frac{1}{2} F(u) \\
& -\alpha A B\left(F(u)^{2}+G(u)^{2}\right) G(u)=0 \\
& 2 \frac{d G(u)}{d u}-\frac{1}{2} G(u) \\
& -\alpha A B\left(F(u)^{2}+G(u)^{2}\right) F(u) \\
& -C_{1} \operatorname{Cos}^{2}\left(\omega_{1} u\right) \\
& +C_{2} \operatorname{Cos}^{2}\left(\omega_{2} u\right) \\
& =0 \\
& \frac{d H(u)}{d u}=\Omega
\end{aligned}
$$

With a $\mathrm{H}(\mathrm{u})$ function of $\mathrm{u}$ adding an extra dimension. It is known that, we need at least 3 dimensions to find chaos in problems involving continuous change described by differential equations [24]. Here $\Omega$ is a constant, $C_{1}$ and $C_{2}$ are the amplitudes of external potential and $\omega_{1}$ and $\omega_{2}$ are its frequencies respectively.

\section{Numerical Results}

Methods from the viewpoint of nonlinear dynamics and chaos theory are quite useful in solving problems where chaos is present. It is difficult to obtain exact solutions directly for the Thirring model with potential. So we present some numerical results to get more information about the quantum dynamics of spinor type instantons in vacuum. We set $\alpha A B=1$ in the paper because of the existence of the spinortype Thirring instantons for this value [13]. Also the fixed points of sytem 
$\left(-\frac{1}{2},-\frac{1}{2}\right)$ and $\left(\frac{1}{2}, \frac{1}{2}\right)$ are used as initial $\begin{aligned} & \text { programming language are benefited in } \\ & \text { the paper. }\end{aligned}$
conditions. TISEAN and Maxima's

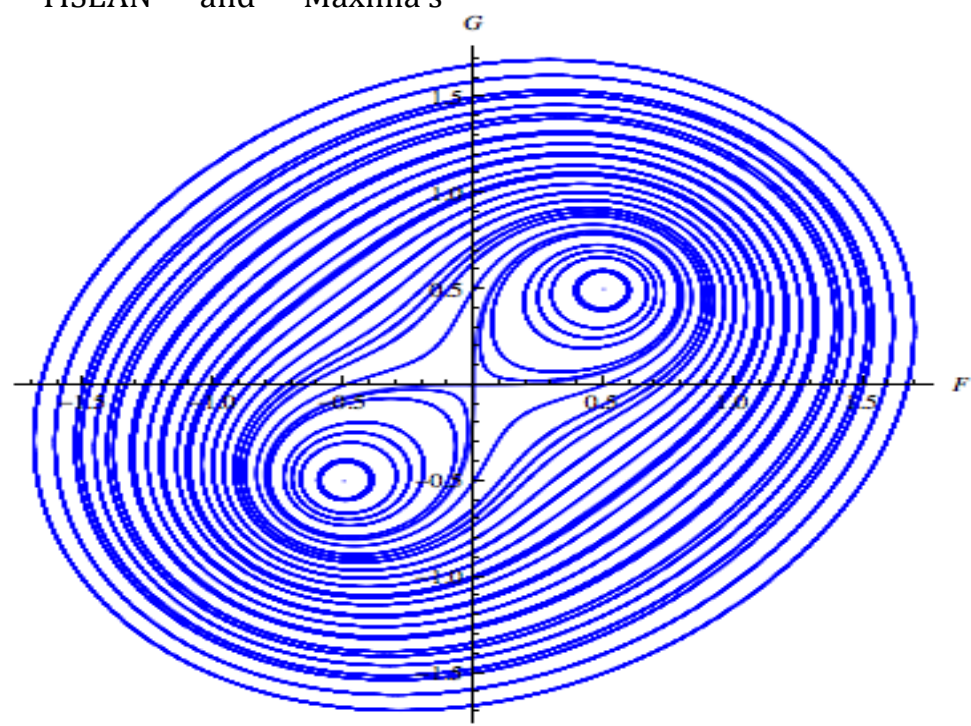

Şekil 1. Undamped Duffing type stability characterization of Thirring instantons for $\alpha A B=1$

In Fig. 1, the phase space display of Thirring instantons is given for without potential. As can be seen from Fig.1, the phase space dynamics of 2D spinor type Thirring instantons possess a Duffing oscillator type steady-characterization without forcing and damping $[14,15]$. It is well known that Duffing oscillator is a famous example describing the motion of a classical particle in a double well potential [25]. In Fig. 2, the phase space displays for different amplitude values are seen. For the weak potential in Fig. 2(a) the system shows regular behaviour. As we reinforce the amplitude, Fig. 2(b) shows a regular island embedded in the chaotic sea. It is interesting that the obtained phase space plot has a typical KAM-stability island. Namely, some originally periodic solutions remain regular while others start to behave chaotically [26]. Due to more amplified potential, Fig. 2(c) exhibit more chaotic regions. Also similar results are obtained for different frequency values as can be seen from Fig. 3. The system exhibits KAM-like behaviour in Fig. 3(b) and more chaotic regions in Fig. 3(c). So we can conclude that external potential having certain frequencies may change the undamped Duffing-type stability characteristics of spinor-type Thirring instantons in phase space for the same initial conditions. Thirring instantons can not maintain its stability for the above initial conditions, when the amplitude of external potential increase enough. 
Fatma AYDOĞMUŞ / The Effect of Bichromatic Potential on Thirring Instantons

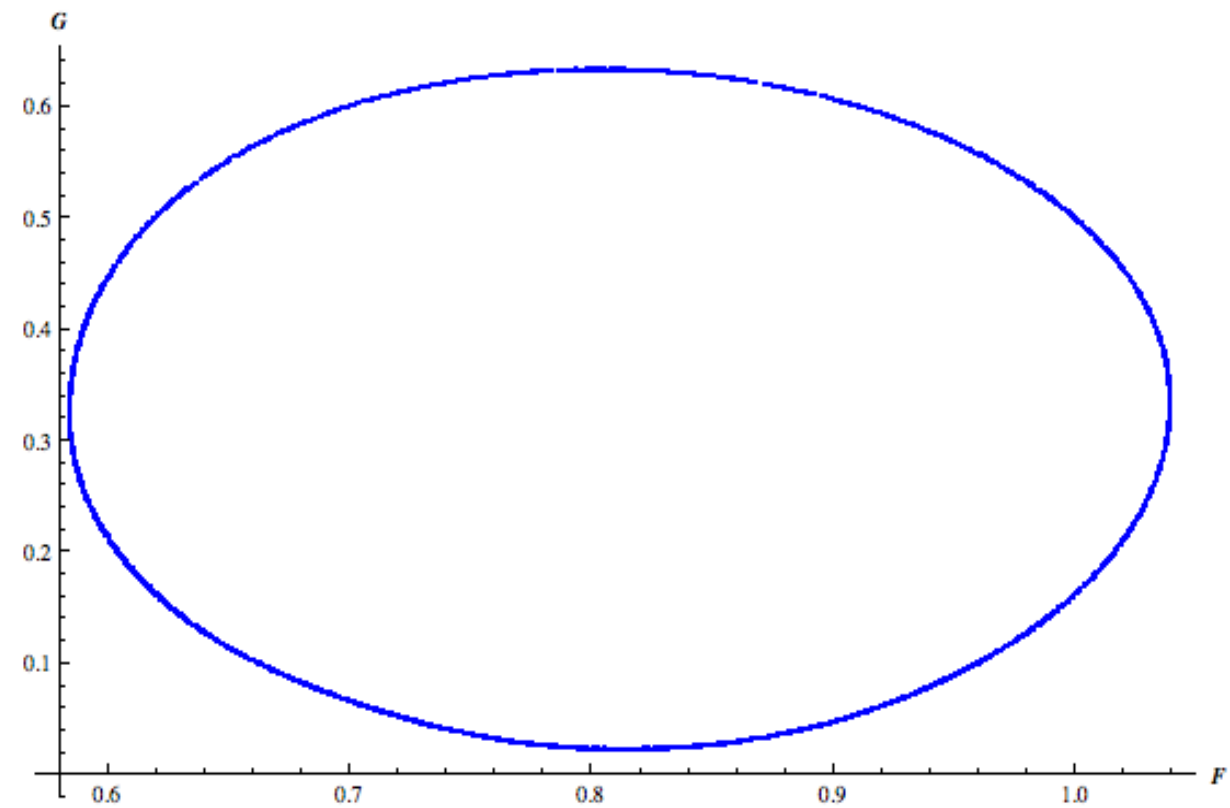

(a)

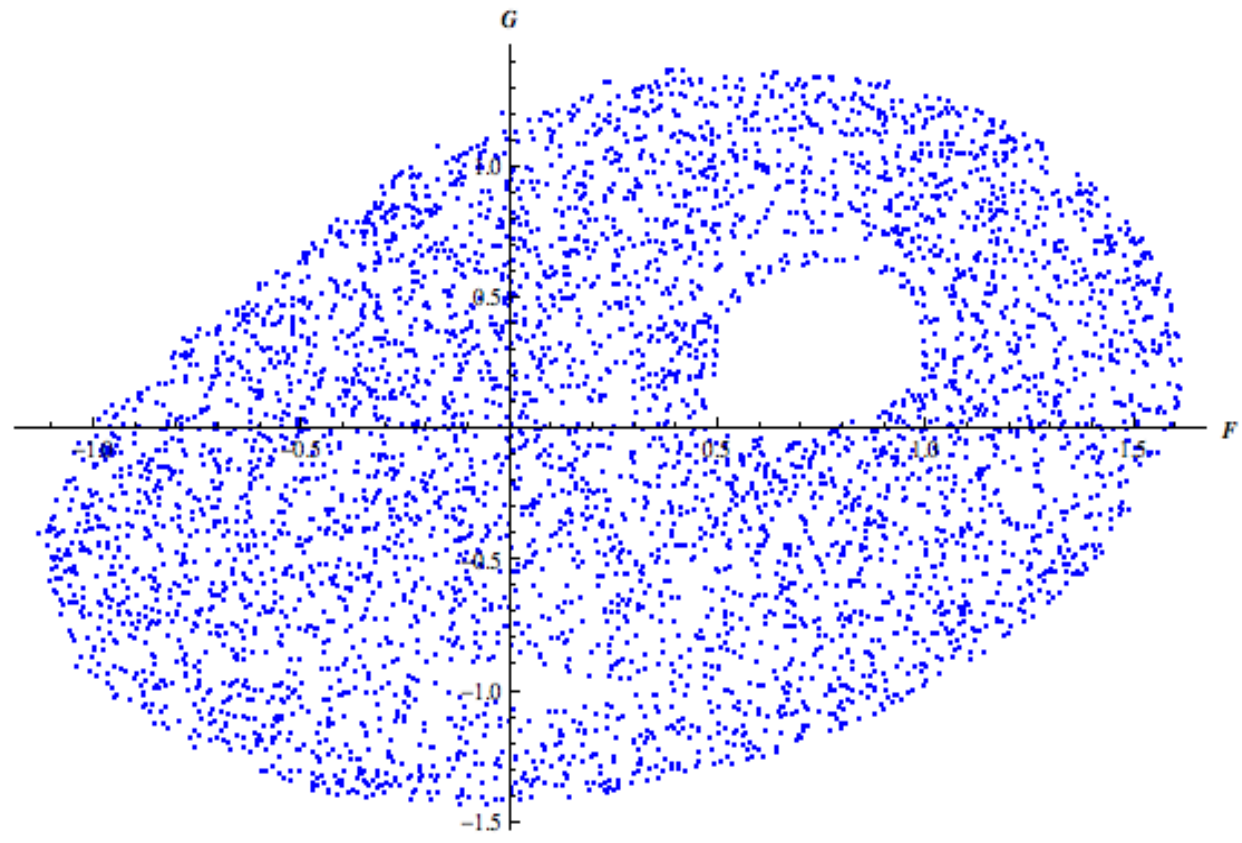

(b) 


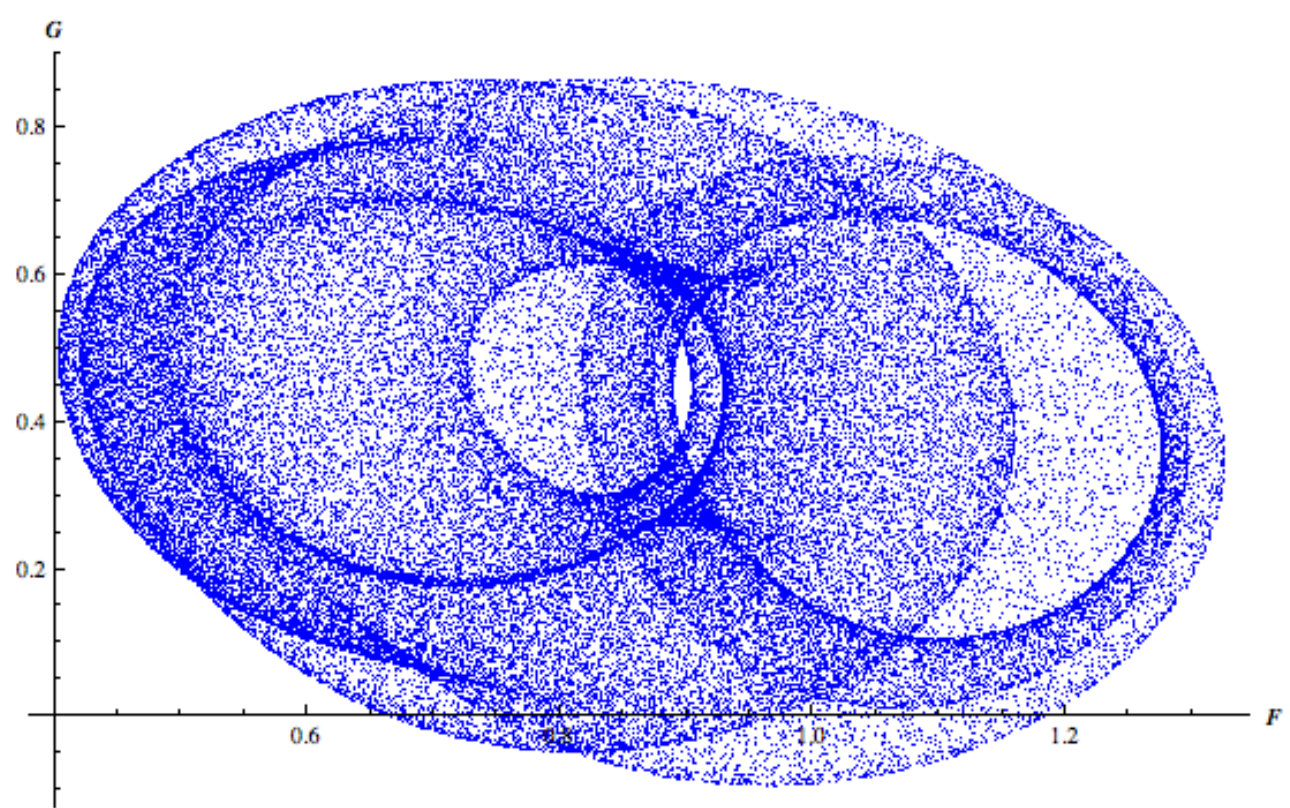

(c)

Şekil 2. Transition to chaos under the bichromatic potential for $\omega_{1}=0.2$ and $\omega_{2}=0.1$ (a) $\mathrm{C}_{1}=0.006, \quad \mathrm{C}_{2}=0.007$ (b) $\mathrm{C}_{1}=0.1, \mathrm{C}_{2}=0.09$, (c) $\mathrm{C}_{1}=0.171, \mathrm{C}_{2}=0.13$

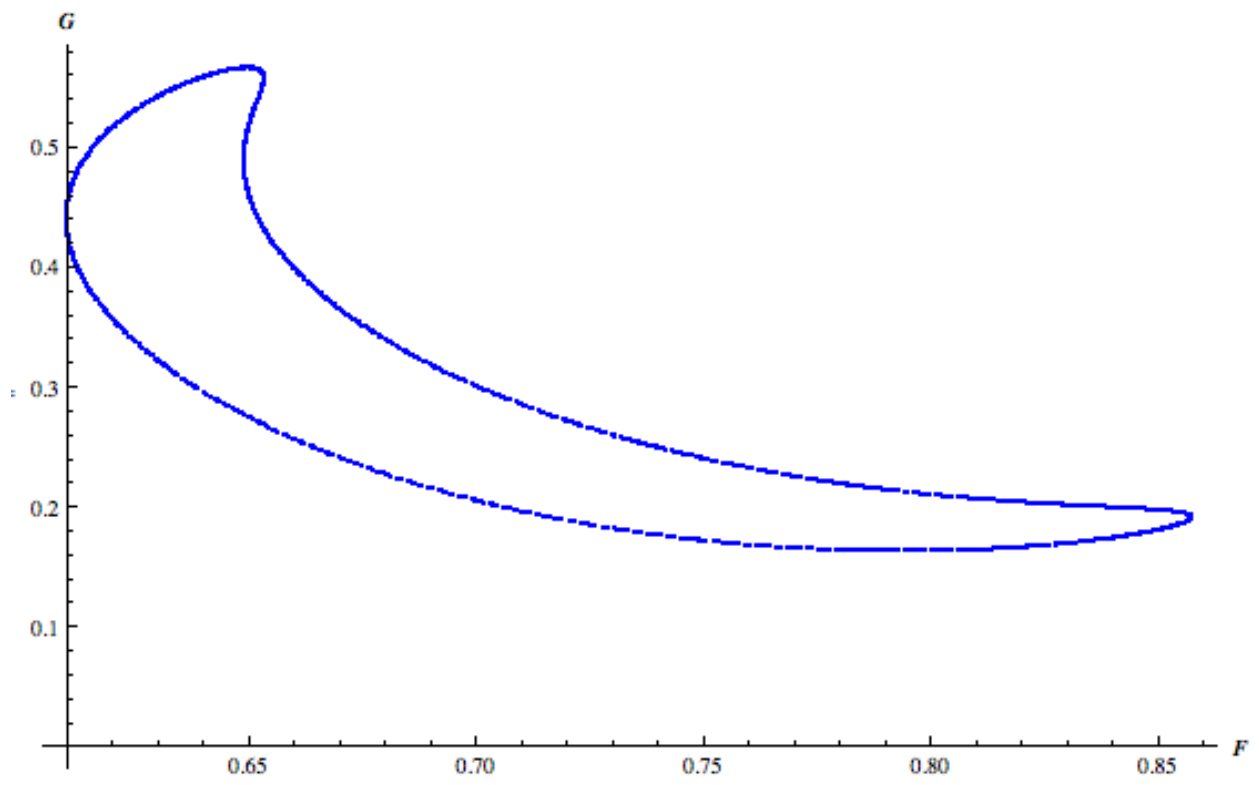

(a) 


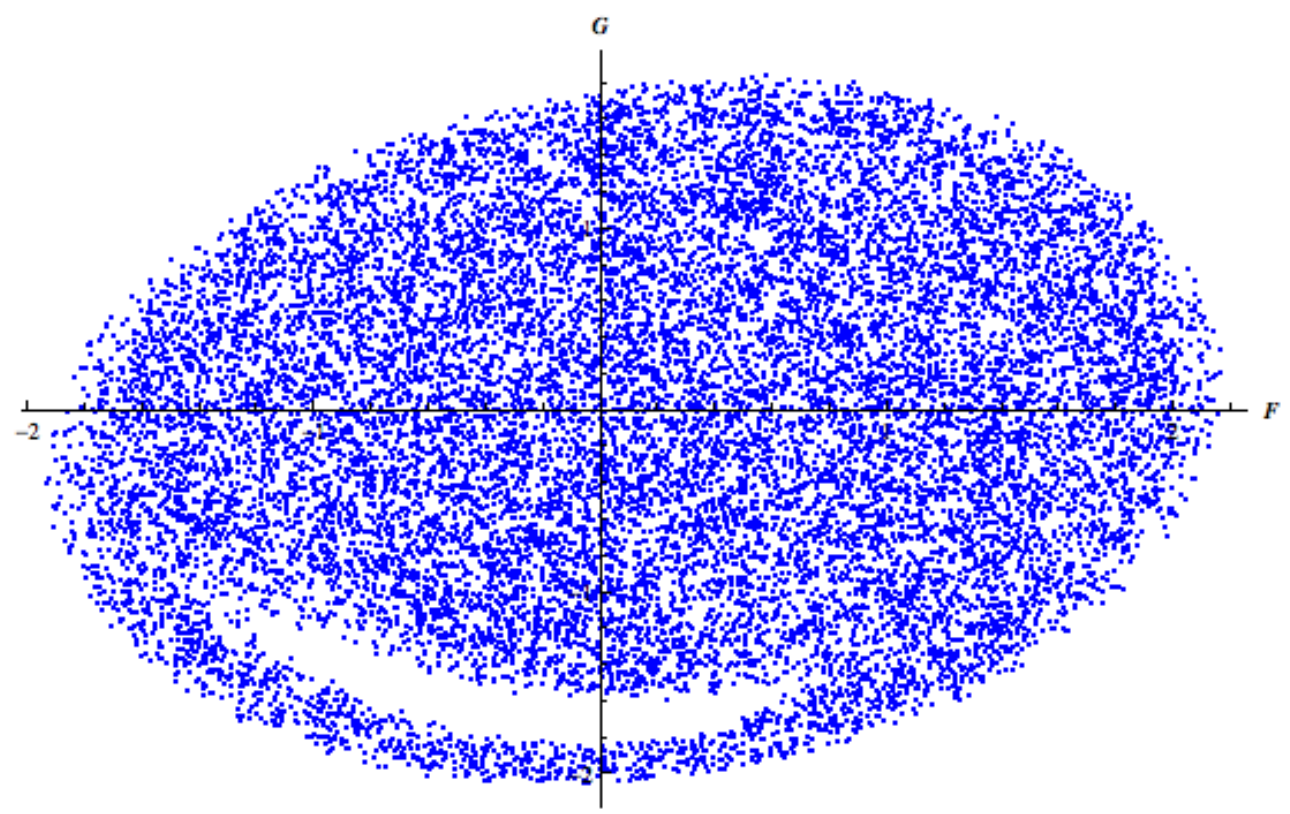

(b)

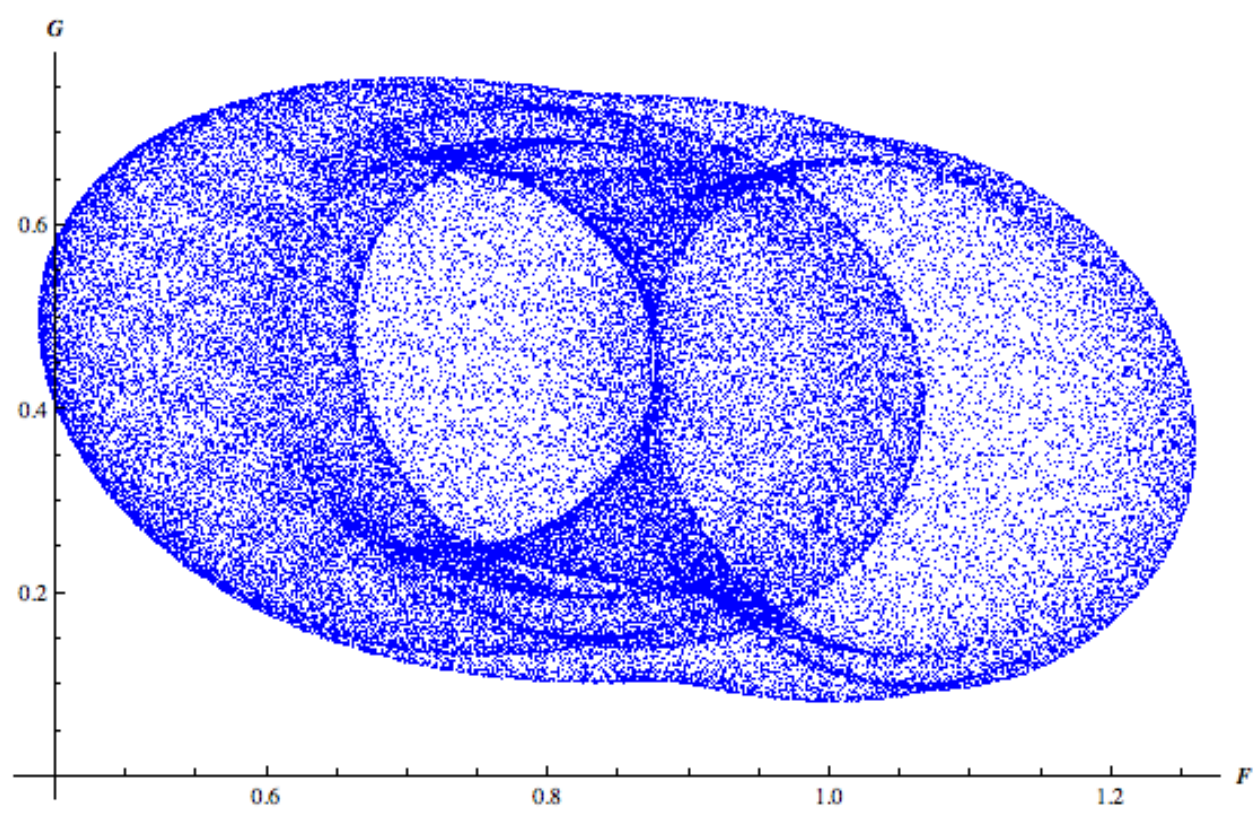

(c)

Şekil 3. Transition to chaos under the bichromatic potential for $\omega_{1}=0.1$ and $\omega_{2}=0.05$ (a) $\mathrm{C}_{1}=0.006, \mathrm{C}_{2}=0.007$ (b) $\mathrm{C}_{1}=0.1, \mathrm{C}_{2}=0.09$, (c) $\mathrm{C}_{1}=0.171, \mathrm{C}_{2}=0.13$ 


\section{Discussion and Conclusion}

It is known that Thirring model contains many of the typical features of the quantization of relativistic quantum field theories. Recently, many studies have been done on this model to understand its dynamical nature [14-16 and 27]. In this paper, we consider the Thirring nonlinear differential equations system formed by the use of Heisenberg ansatz and we investigate it under the bicromatical potential to understand how the behaviours of spinor type Thirring instantons could be affected. In the view of obtained results, we can conclude that the undamped Duffing-type stability characteristics of spinor-type Thirring instantons disappear depending on the external potential parameter values. The bichromatic potential destroys the regularity of system and generates a stochastic layer. Thirring instantons lies on this layer which correspond to irregular curves with potential, i.e., a number of chaotic instantons appears depending on the external potential parameter values [28-29].

\section{References}

[1] Louis de Broglie, An Introduction to the Study of Wave Mechanics, London, Methuen and Co.Ltd, 1930.

[2] M. Dunajski, Solitons, Instantons, and Twistors, Oxford University Press, New York 2010.

[3] C. Rebbi and G. Solliani, Solitons and Particles, World Scientific, 1984; T. Dauoxois and M. Peyrard, Physics of Solitons, Cambridge University Press, 2006.

[4] C.Houghton, Instantons, in the Encyclopaedia of Nonlinear Science, Routledge, New York, 2005.
[5] ] R. Rajaraman, Solitons and Instantons, North-Holland, Elsevier Science Publisher, 1982.

[6] M. Kuhlen, QCD at HERA: The Hadronic Final State in Deep Inelastic Scattering, SpringerVarlag Berlin, 1998.

[7] M. Eto and M. Nitta, Semilocal fractional instantons, Journal of High Energy Physics, 67, 2016, pp.1-19

[8] S. Moch, A. Ringwald, and F. Schrempp, Instantons in Deepinelastic Scattering: The Simplest Process, Nucl. Phys., B507, 1997, pp.134-156

[9] A. Ringwald and F. Schrempp, Instanton-induced Cross-sections in Deep-inelastic Scattering, Phys. Lett.,B438, 1998, pp.217-228.

[10] F.Schrempp and A. Ringwald, Zooming-in on Instantons at HERA, Phys.Lett. B503, 2001, pp.331-340.

[11] A. Ringwald and F. Schrempp, Qcdins2.0: A Montecarlo Generator for Instanton induced Processes in Deep-inelastic Scattering, Comput. Phys. Commun., 132, 2000, pp. 267305.

[12] F. Kortel, On some solutions of Gursey's conformal-invariant spinor wave equation, Il Nuovo Cimento, 4, 1956, pp.210-215

[13] K.G. Akdeniz and A. Smailagic, Classical Solitons for Fermionic Models, Il Nuovo Cimento, 51A, 1979, pp.345-357.

[14] F. Aydogmus, The Behaviours of Spinor Type Instanton Attractors in Phase Space, Istanbul University, Institute of Science, Physics Department Ph.D. Thesis, 2012).

[15] B. Canbaz, C. Onem, F. Aydogmus and K. G. Akdeniz, From Heisenberg Ansatz to Attractor of Thirring Instanton, Chaos Solitons\& Fractals, 45, 2012, pp. 188-191. 
[16] F. Aydogmus and E. Tosyali, Common Behaviours of Spinor Type Instantons in 2-D Thirring and 4-D Gursey Fermionic Models, Advances in High Energy Physics, 148375 2014, pp.1-11

[17] F. Bruckmann, Instanton Constituents in the $\mathrm{O}(3)$ Model at Finite Temeperature, Phys. Rev. Lett. 100, 051602, 2008, pp.1-4.

[18] W. Brendel, F. Bruckmann, L. Janssen, A. Wipf and C. Wozar, Instanton Constituents and Fermionic Zero Modes in Twisted CP(n) Models, Phys. Lett. B 676, 116, 2009, pp.116-125.

[19] M. Nitta and W. Vinci, Decomposing Instantons in Two Dimensions, J. Phys. A, 45, 17, 2012, pp.1-23.

[20] G. V. Dunne and M. Unsal, Uniform WKB, Multi-instantons and Resurgent Trans-Series, Phys. Rev.D 89, 105009, 2014, pp.1-22.

[21] M. Nitta, Fractional Instantons and Bions in the $\mathrm{O}(\mathrm{N})$ Model with Twisted Boundary Conditions, Journal of High Energy Physics 37, 108, 2015, pp.1-37.

[22] W. E. Thirring, A Soluble Relativistic Field Theory, Anal Physics, 3, 1958, pp. 91-112).

[23] W. Heisenberg, Zeits. F. Naturf. A9,1954, 292

[24] S. Strogatz, Nonlinear Dynamics and Chaos:With Applications to Physics, Biology, Chemistry, and Engineering, PerseusBooks, 1999.

[25] G. Duffing, Erzwungene Schwingungenbei Veranderlicher Eigenfrequenz., F. Vieweg u. Sohn, Braunschweig, 1918.

[26] A.N. Kolmogorov, On the Conservation of Conditionally Periodic Motions under Small Perturbation of the Hamiltonian, Dokl. akad. nauk SSSR, vol. 98, 1954, pp.527-530, Engl.10 transl.: Stochastic Behaviour in Classical and Quantum Hamiltonian Systems, Volta Memorial conference, 1977, Lecture Notes in Physics, 93, Springer, 1979, pp.51-56, Arnold, V. I., Russ. Math. Surv., 18, 13, 1963, Moser, J., Nachr. Akad. Wiss. Gött. II, 1, 1962.

[27] F. Aydogmus and E. Tosyali, Numerical Analysis of Thirring Model under White Noise, Journal of Physics: Conference Series 633, 012022, 2015, pp.1-4

[28] F. Aydogmus, Chaos in a 4D Dissipative Nonlinear Fermionic Model, Journal of Modern Physics C, 26, 7, 1550083, 2015, pp.1-13.

[29] F. Aydogmus, Unstable Behaviours of Classical Solutions in Spinor-type Conformal Invariant Fermionic Models, arXiv:1508.00610 [hep-th], 2015 